\title{
Study of clinical and hematological profile in children with dengue fever in a teaching hospital in Telangana
}

\author{
Ramana Sastry C.P.V. ${ }^{1}$, Padmavathi M. ${ }^{2}$ \\ ${ }^{1}$ Dr. C. P. V. Ramana Sastry, Associate Professor, ${ }^{2}$ Dr. Maram Padmavathi, Assistant Professor; both authors are \\ affiliated with Department of Paediatrics Malla Reddy Medical College for Women, Telangana, Andra Pradesh, India. \\ Corresponding Author: Dr. Maram Padmavathi, Assistant Professor, Department of Paediatrics Malla Reddy Medical \\ College for Women, Telangana, Andra Pradesh, India.
}

\begin{abstract}
Introduction: The aim of the study was to determine the clinical and hematological profile in patients with Dengue fever. Materials and Methods: This was a prospective study done at Malla Reddy Medical College for Women, Telangana, for a period of six months. A total of 110 cases of dengue infection were studied. The patient demographics, clinical symptoms, hematological characteristics such as hemoglobin, total leucocyte count, platelet count, serological studies and abdominal ultrasound findings were noted. Results: In the present study, 85/110 (77.2\%) cases were of dengue fever, 20/110 (18.1\%) were dengue hemorrhagic fever (DHF) and 05/110 (4.5\%) were of dengue shock syndrome (DSS). Patient age ranged from 0- 12 years. Majority, 55/110 (50\%) were among 6-8 years. Males were affected more than females (59\% boys and $41 \%$ were girls). Moderate thrombocytopenia was seen in $54.5 \%$ of cases and raised hematocrit $(>47 \%)$ was observed in $22.7 \%$ cases. Most of the cases $75(68.1 \%)$ were noted in September and October. Conclusion: Dengue is a common viral infection which may have serious consequences especially in children. Simple haematological parameters along with serological tests and ultrasound of abdomen are helpful in the diagnosis and appropriate management of these patients.
\end{abstract}

Keywords: Dengue fever in children,Platelet count, Thrombocytopenia, NS1Ag

\section{Introduction}

Dengue infection is an emerging disease of international concern and is a major health problem in India. Globally the incidence of dengue has increased in the recent years. The WHO estimates that presently about two fifths of the world population is at risk for this viral infection [1]. Dengue fever was first reported by Benjamin Rush in 1780 as "break bone fever." It is a mosquito borne viral infection with four serotypes. It commonly presents as dengue without warning signs, dengue with warning signs and severe dengue [2].

Dengue infection is frequently confounded with other febrile illnesses (OFI), presenting with non-specific clinical symptoms and clinical features analogous to OFI. During the early stages of dengue, the presence of non-specific febrile illness makes precise diagnosis very difficult, resulting in inefficient treatment thereby increasing the morbidity and mortality of this disease $[3,4]$. Severe dengue fever, if not appropriately

Manuscript received: $10^{\text {th }}$ January 2019

Reviewed: $20^{\text {th }}$ January 2019

Author Corrected: $26^{\text {th }}$ January 2019

Accepted for Publication: 31 $31^{\text {st }}$ January 2019 managed, may lead to rapid death, particularly in children [5,6]. Dengue is an acute febrile disease caused by a flavivirus with four known serotypes (DENV-1, DENV-2, DENV-3, and DENV-4). The four serotypes can lead to variable clinical presentations, ranging from asymptomatic to severe forms [7].

Children represent more peculiar characteristics since dengue diagnosis, and recognition of severe forms are both more difficult to find than in adults. The common signs and symptoms are fever, headache, myalgia, arthralgia, and bleeding manifestations. The exact clinical profile is necessary for the patient's management and hence, crucial for saving life [8].

Aim of the study: To study clinical and hematological profile in children with dengue fever.

\section{Materials and Methods}

No ethical issues were involved in the study. This was a prospective observational study done in the department of Paediatrics at Malla Reddy Medical College for 


\section{Original Research Article}

Women,Telangana over a period of 6 months from July 2018 to January 2019. Blood samples were collected in EDTA anticoagulant for hematological investigations, and in plain tubes/vacutainers for microbiological and biochemical investigations. No surgical interventions were done in any of the patients. Statistical analysis was done by taking the percentage of the cases. All the suspected cases of dengue etiology who presented with fever and with or without rashwere admitted in the department of pediatrics.

\section{Inclusion criteria}

1. Age group of 0 to 12 years

2. Both genders

3. Patients with NS1 Ag positive

4. Serologically confirmed IgM positive dengue fever

\section{Exclusion criteria}

1. Age group $>12$ years

2. Preexisting chronic diseases
Complete clinical history was taken from parents of children admitted in pediatric ward. Local and systemic examination in all cases was done thoroughly.

All the routine investigations were asked such as complete blood count (CBC), peripheral blood smear examination, random blood sugar, complete urine analysis, microbiological investigations included NS1 dengue serology.

Dengue serology was done by ELISA method in all the cases. Ultrasonography of abdomen was done in all the cases.

For routine investigations venous blood was collected from cubital vein from all patients admitted with symptoms suggestive of dengue fever.

Hematological parameters were evaluated including hemoglobin percentage, hematocrit, platelet count, total leucocyte count, differential leucocyte count.

\section{Results}

A total of 110 children were included in the present study during period of 6 months. In the present study, 77.2\% (85/110) cases were of dengue fever, $18.1 \%$ (20/110) were dengue hemorrhagic fever and $4.5 \%$ (5/110) were of dengue shock syndrome.

Table-1: Age-wise distribution of cases.

\begin{tabular}{|c|c|c|}
\hline Age & No. of cases & Percent (\%) \\
\hline $0-2$ years & 05 & $4.5 \%$ \\
\hline 3-5 years & 15 & $13.6 \%$ \\
\hline 6-8 years & 55 & $50 \%$ \\
\hline 9-11 years & 27 & $24.5 \%$ \\
\hline 11-12 years & 08 & $7.2 \%$ \\
\hline Total & 110 & $100 \%$ \\
\hline
\end{tabular}

In the present study, age group distribution included from 0 to12 years. Majority of the cases were among 6-8 years age.

Gender-wise distribution of cases: in the present study, there were 65 (59\%) boys and $45(41 \%)$ girls and the male to female ratio was 1.4:1.

Table-2: Clinical symptoms of the cases.

\begin{tabular}{|c|c|c|}
\hline Clinical Symptoms & No. of cases & Percent (\%) \\
\hline Fever & 110 & $100 \%$ \\
\hline Myalgia & 70 & $63.6 \%$ \\
\hline Fever and myalgia & 90 & $81.8 \%$ \\
\hline Fever with rash & 20 & $18.1 \%$ \\
\hline Petechiae & 20 & $18.1 \%$ \\
\hline Abdominal pain & 45 & $40.9 \%$ \\
\hline Nausea and vomiting & 70 & $63.6 \%$ \\
\hline Ascites & 10 & $9.09 \%$ \\
\hline Shock & 02 & $1.8 \%$ \\
\hline
\end{tabular}

In the present study, fever was present in all $100 \%$ of the cases. 


\section{Hematological Parameters}

Table-3: Hemoglobin range.

\begin{tabular}{|c|c|c|}
\hline Hb & No. of cases & Percent $(\%)$ \\
\hline$<5 \mathrm{gm} \%$ & 04 & $3.6 \%$ \\
\hline $5-10 \mathrm{gm} \%$ & 40 & $36.3 \%$ \\
\hline $10-15 \mathrm{gm} \%$ & 56 & $50.9 \%$ \\
\hline$>15 \mathrm{gm} \%$ & 10 & $9.0 \%$ \\
\hline Total & $\mathbf{1 1 0}$ & $\mathbf{1 0 0} \%$ \\
\hline
\end{tabular}

Present study showed hemoglobin range from less than $5 \mathrm{gm} \%$ to more than $15 \mathrm{gm} \%$. Almost half (50.9\%) cases showed hemoglobin value between 10-1 $5 \mathrm{gm} \%$.

Table-4: Hematocrit value.

\begin{tabular}{|c|c|c|}
\hline Hematocrit & No. of cases & Percent $(\%)$ \\
\hline $26-36 \%$ & 05 & $4.5 \%$ \\
\hline $37-47 \%$ & 80 & $72.7 \%$ \\
\hline$>47 \%$ & 25 & $22.7 \%$ \\
\hline Total & $\mathbf{1 1 0}$ & $\mathbf{1 0 0} \%$ \\
\hline
\end{tabular}

Raised hematocrit $(>47 \%)$ was observed in $22.7 \%$ cases $(25 / 110)$.

Table-5: Total leukocyte count and platelet count.

\begin{tabular}{|c|c|c|c|c|c|}
\hline TLC/ cumm & No. of cases & Percent $(\%)$ & Platelet count/cumm & No. of cases & Percent $(\%)$ \\
\hline$<4000$ & 20 & $18.1 \%$ & $<20000$ & 15 & $13.6 \%$ \\
\cline { 3 - 6 } & & & $20000-50000$ & 60 & $54.5 \%$ \\
\hline $4000-11000$ & 85 & $77.2 \%$ & $50000-1.4$ lakhs & 25 & $22.7 \%$ \\
\hline$>11000$ & 05 & $4.5 \%$ & $>1.4$ lakhs & 10 & $9.0 \%$ \\
\hline Total & $\mathbf{1 1 0}$ & $\mathbf{1 0 0} \%$ & Total & $\mathbf{1 1 0}$ & $\mathbf{1 0 0} \%$ \\
\hline
\end{tabular}

Total WBC count was within normal limits (4000-11000/cumm) in 77.2\% cases. Moderate thrombocytopenia (50000$1.4 \mathrm{lakh} / \mathrm{cumm})$, was seen in $22.7 \%$ (25/110) and severe thrombocytopenia was seen in $13.6 \%(15 / 110)$ cases.

Liver enzymes were elevated in 60 (54.5\%) cases and were normal in $50(45.4 \%)$ cases.

USG findings: Ultrasound of the abdomen showed hepatomegaly in 60 (54.5\%) of the cases, ascites in $18.1 \%$ and pleural effusion in $18.1 \%$ cases.

NS1 Ag: NS 1 antigen test was positive in $90 \%$ of cases, serum dengue IgM in $60 \%$ and dengue IgG was positive in $40 \%$ of cases.

Seasonal Variation: Most of the cases 75 (68.1\%) were noted in September and October months and 35 (31.8\%) cases were recorded in November and December.

\section{Discussion}

In the present study, 85/110 (77.2\%) cases were of dengue fever,20/110 (18.1\%) were dengue hemorrhagic fever (DHF) and 05/110 (4.5\%) were of dengue shock syndrome (DSS). In the study by Mishra et al[9] the total number of cases was 97 , out of which 84 were cases of non-severe dengue (undifferentiated fever, dengue fever withwarning signs, and dengue fever without warning signs) and 13 were cases of severe 
dengue (DHF and DSS). Srinivasa et al[10] studied 200 confirmed dengue cases. Jain et al [11] studieda total of 65 children in which as per WHO classification, 42 (64\%) cases belonged to dengue with warning signs, 8 cases had severe dengue and 15 cases had dengue without warning signs.

Kumaret al [12] studied a total of 306 children admitted with a diagnosis of dengue fever during their study period. Alam et al[13]in their study observed that over $40 \%$ of the patients presented with dengue fever, $27.8 \%$ had DHF grade-1, 16.7\% DHF grade-2, 3.7\% grade-3 and $11.1 \%$ grade- 4 .

In the present study, age group for dengue fever studied was from 0- 12 years. Majority of the patients, almost $50 \%$ were among 6-8 years, next common age group was among 9-11 years having $24.5 \%$ cases, $3.6 \%$ $(15 / 110)$ in the age group of $3-5$ years, and $4.5 \%$ $(5 / 110)$ were infants.

Mishra et al[9]in their study observed that the mean age of hospitalized patients was 8.7 years. $63.9 \%$ of patients were admitted in the hospital for 3-6 days. Seven children out of 13 severe dengue patients were admitted for more than 6 days. The mean tenure of hospitalization was 3.8 days.

In the study by Alam et al [13]the age ranged from 6 months to 15 years with a mean of $6.5 \pm 3.5$ years and about half $(51.9 \%)$ of them were between 5-10 years age.

In the study by Srinivasa et al [10] the most common affected age group was that of school going children $(42 \%)$ and adolescents $(36 \%)$.

In the study by Kumar et al[12] majority 175 (57\%) were in the age group of 6-12 years, $84(27 \%)$ in the age group of $1-5$ years, and $47(16 \%)$ were infants. The mean age of the study population was estimated to be $7.8 \pm 3.2$ years. In the study by Jain et al[11] the most common age group was between 5 to 10 years $(46 \%)$ followed by $1-5$ year age group. Infants were only $7.6 \%$ and the youngest one was 7 months old. Our findings compare well with the above observations.

In the present study, majority of the patients were boys $59 \%(65 / 110)$ compared to girls $40.9 \%(45 / 110)$. In the study by Mishra et al[9] there were $75(77.31 \%)$ males and $22(22.68 \%)$ females. For both the groups of severe and non-severe dengue, males had higher incidence. The male-tofemale ratio was 3.4:1. The male to female ratio was $1.1: 1$, and $1: 1$ in the studies by Srinivasa et al
[10] and Alam et al respectively.[13]. In the study by Jain et al [11]35 (53\%) were boys and 30 (47\%) were girls. Male to female ratio was 1.16:1. Similarly, in the study by Kumaret al ${ }^{[12]}$ males were more in number (158) than females (148) with a male: female ratio of 1.06:1.

Seasonal distribution: In our study, most of the cases $75(68.1 \%)$ were recorded in the month of September and October and slightly less number, 35(31.8\%) were seen in November and December. Mishra et al [9] observed that most of their cases were admitted in the rainy and winter seasons between the months of July and November. The peak of admission was seen in the month of September with 59 cases $(60.8 \%)$. The least admissions were seeninthe summer season, specifically in the month of April, consisting of 4 cases (4.1\%).

Clinical features: In the present study, fever was present in $100 \%$ of the cases; myalgia $(63.6 \%)$, fever with myalgia in $81.8 \%$ and fever with rash in $18.1 \%$.

Mishra et al [9] also observed fever in $100 \%$ of the cases, myalgia in $76.8 \%$ and abdominal pain in $54.3 \%$ cases. The most common bleeding manifestations in both severe and non-severedengue were petechiae seen in $22.1 \%$ cases.

Srinivasa et al[10] studied 200 cases andobservedthat all the cases all had fever (100\%),144 (72\%) had vomiting, 92(46\%) had abdominal pain, 67(33.5\%) had hepatomegaly, $21(10.5 \%)$ had rashes, 4(2\%) had splenomegaly, 26(13\%) had bradycardia and 47(23.5\%) developed hypotension and shock. The mostcommon bleeding manifestation was petechiae $(6.2 \%)$ followed by hemetemesis in $4.1 \%$ and epistaxis in $3.6 \%$ cases.

Jain et al [11] observed fever in all dengue patients with mean duration of 5.6 days. The common presentation by these children included headache $(64 \%)$, myalgia $(63 \%)$, bleeding $(58 \%)$ and decreased urine output $(53 \%)$

Among the clinical findings, hepatomegaly and splenomegaly were noted in $90 \%$ and $26 \%$ of the cases respectively. Clinical fluid accumulation in form of ascites and pleural effusion with reduced air entry were observed in $40 \%$ and $43 \%$ of cases.

Kumar et al [12 ]observed fever in all of their patients, with the duration of hospital stay $<5$ days in 205 (72\%) and 5-10 days in 75 patients (25\%). The most common symptoms were vomiting in $168(54.90 \%)$, abdominal pain in $111(36.3 \%)$, bleeding manifestation in 
$43(14.05 \%)$, headache in $41(13.40 \%)$, myalgia in 32 $(10.5 \%)$, and lethargy $(9.8 \%)$. The other symptoms observed in their study were arthralgia (9.5\%), altered sensorium (7.8\%), rash (7.2\%), diarrhea (6.5\%), oliguria $(6.5 \%)$, cough/rhinitis $(5.2 \%)$, anorexia (3.9\%), and retro-orbital pain (1.3\%).

Alam et al[13] observed fever $>5$ days duration in $63 \%$ children with continued type of fever beingpredominant $(75.9 \%)$. About $60 \%$ of patients had abdominal pain, $57 \%$ vomiting, $46.3 \%$ myalgia, $31.5 \%$ had headache, $18.5 \%$ arthralgia, $14.8 \%$ retro-orbital pain, $9.3 \%$ loose stool and $3.7 \%$ had runny nose/cough.

Haematology findings: The present study observed hemoglobin range of less than $5 \mathrm{gm} \%$ to more than 15 $\mathrm{gm} \%$. Hemoglobin of $5-10 \mathrm{gm} \%$ was seen in $36.3 \%$ cases and $10-15 \mathrm{gm} \%$ range was seen in $50.9 \%$ cases. Raised hematocrit ( $>47 \%$ ) was observed in $22.7 \%$ cases $(25 / 110)$.

The total WBC count was within normal limits (4000$11000 /$ cumm) in $77.2 \%$ cases and leucopenia of less than 4000 cells/cumm was seen in $18.1 \%$ (20/110) cases. Only $4.5 \%$ cases had leukocytosis with more than 11000/cumm count. Mild, moderate and severe thrombocytopenia were seen in $22.7 \%, 54.5 \%$ and $13.6 \%$ cases respectively.

Mishra et al[9] in their study reported that $58.76 \%$ of the cases had normal leukocyte count, while leucopoenia was seen in $25.77 \%$ and leukocytosis in $15.46 \%$ of the cases. Among the liver enzymes, SGOT was elevated in a larger proportion $(47.42 \%)$ of patients when compared to alanine aminotransferase (SGPT) which was $30.92 \%$.

According to the study by Srinivasa et al [10]out of 200 cases, 194(97\%) had thrombocytopenia, 189(94.5\%) had hemoconcentration, 126(63\%) had leucopenia< 4000/cumm and 83(41.5\%) had raised liver enzymes Jain et al [11] observed evidence of raised hematocrit $(>35 \%)$ in $84 \%$ of cases.

Thrombocytopenia ( $<1$ lakh) was observed in $80 \%$ of cases with $10 \%$ of patients having platelet count $<20,000 / \mathrm{mm}$, most of the cases had platelet count between 50,000 and 1-lakh. Leucopenia $(<5000)$ was observed in $44 \%$ of cases. Abnormal liver enzymes were observed in $38 \%$ of patients. Kumaret al[12] observed $14.1 \%$ of their patients had a platelet count $<20,000$ and low platelet count had significantly correlated with the severity of dengue. The mean platelet recovery time was $2-5$ days.
The liver enzymes aspartate aminotransferase (AST) and alanine aminotransferase (ALT) were elevated significantly. Prolonged activated partial thromboplastin time (aPTT) was seen in $102(33.3 \%)$ children while 64 $(20.9 \%)$ had elevated prothrombin time.

In the study by Alam et al [13] about one-third (32\%) of the patients had positive tourniquet test. Five (9.3\%) had low WBC count. Onethird (33\%) of patients had platelet count, 51000 to 1 lakh, $25.9 \%$ had between 21000 to 50000 and $9.3 \%$ had $<20000$ platelet count and $31.5 \%$ patients had platelet count $>1$ lakh.

All children exhibiting a packed cell volume (PCV) of less than $45 \%$ and over $40 \%$ had raised serum alanine aminotransferase (ALT).

Comparative studies based on USG: In the present study, Ultrasound of the abdomen showed hepatomegaly in $60(54.5 \%)$ of the cases, followed by ascites in $18.1 \%$ cases and pleural effusion in $18.1 \%$ cases.

Mishra et al [9] in their study observed that the Ultrasound of the abdomen detected hepatomegaly in $52.75 \%$ of the cases, which was the most common finding followed by ascites $(25.77 \%)$ and gall bladder wall edema in $2.06 \%$ cases.

Srinivasa et al [10] also reported the ultrasonographic findings of hepatomegaly in $84(42 \%)$, splenomegaly in 33 , gall bladder wall thickening in61, pleural effusion in 93 and ascites in 74 cases.

Jain et al [11]observed $(n=31)$ the most common USG finding to be gall bladder edema $(84 \%)$, pleural effusion $(70 \%)$, and ascites $(78 \%)$.

NS 1 Ag and serology: In the present study, NS 1 antigen test was positive in $90 \%$ of cases, $\operatorname{IgM}$ in $60 \%$ and dengue $\mathrm{IgG}$ was positive in $40 \%$ of cases.

Alam et al[13] observed $\operatorname{IgM}$ and $\operatorname{IgG}$ antibodies for dengue virus were positive in $40.7 \%$ and $24.1 \%$ of their patients respectively, both $\operatorname{IgG}$ and IgM were positive $31.5 \%$ cases and serological tests were negative in $3.7 \%$ cases.

In the study by Mishra et al [9] the majority of the patients were positive for NS1 followed by $\operatorname{IgM}$ as a large number of patients presented within 4 days of fever. Serum IgG was estimated in those children who presented with history of 7 days. Tourniquet test was found to be negative in majorityof the patients. 


\section{Conclusion}

Dengue is a common viral infection which may have serious consequences especially in children.

Simple haematological parameters along with serological tests and ultrasound of abdomen are helpful in the diagnosis and appropriate management of these patients.

Funding: Nil, Conflict of interest: None initiated, Perission from IRB: Yes

\section{References}

1. World Health Organization; Dengue and Dengue Hemorrhagic fever. Available in www.who.int/media centre/factsheets./ fs117/en/.

2. Guzmán MG, Kourí G. Dengue: an update. Lancet Infect Dis. 2002 Jan;2(1):33-42.

3. Tanner L, Schreiber M, Low JG, et al. Decision tree algorithms predict the diagnosis and outcome of dengue fever in the early phase of illness. PLoSNegl Trop Dis. 2008 Mar 12;2(3):e196. doi: 10.1371/journal.pntd. 0000196.

4. Manock SR, Jacobsen KH, de Bravo NB, et al. Etiology of acute undifferentiated febrile illness in the Amazon basin of Ecuador. Am J Trop Med Hyg. 2009 Jul; 81(1):146-51.

5. Huy R, Buchy P, Conan A, et al. National dengue surveillance in Cambodia 1980-2008: epidemiological and virological trends and the impact of vector control. Bull World Health Organ. 2010 Sep 1;88(9):650-7. doi: 10.2471/BLT.09.073908. Epub 2010 Apr 7.
6. Sam SS, Omar SF, Teoh BT, et al. Review of Dengue hemorrhagic fever fatal cases seen among adults: a retrospective study. PLoSNegl Trop Dis. 2013 May 2;7 (5):e2194.doi:10.1371/journal.pntd.0002194. Print 2013

7. Sicuro Correa L, Hokerberg YHM, Oliveira RdVCd, Barros DMD. S, Alexandria HAF, Daumas RP, et al. Use of Warning Signs for Dengue by Pediatric Health Care Staff in Brazil. PLoS ONE 2016;11(10): e 0163946.

8. Potts J A, et al. 2010 Prediction of dengue disease severity among Thai pediatric patients using early clinical laboratory indicators PLoSNegl. Trop.Dis.4 1-7

9. Mishra S, Ramanathan R, Agarwalla SK. Clinical Profile of Dengue Fever in Children: A Study from Southern Odisha, India. Scientifica 2016, Article ID 6391594, 6 pages.

10. Srinivasa $\mathrm{S}$, Nawab T, NairCC. Clinical profile and ultrasonographic findings in children with dengue fever.CurrPediatr Res 2014;18 (2):87-90.

11. Jain H. Clinical profile and outcome of dengue fever in hospitalized children of South Rajasthan, India. Int J Contemp Pediatr. 2016;3(2):546-549.

12. Kumar BV,Simna L, Kalpana D, Kailas L. Clinical profile and outcome of children admitted with dengue fever in a tertiary care hospital in South India. Indian J Child Health 2018;5(1);32

13. Alam AS, Sadat SA, Swapan Z, Ahmed AU, Karim MdK, Paul HK, et al. Clinical profile of dengue fever in children. Bangladesh J Child Health 2009;33(2):55-58.

\section{How to cite this article?}

Ramana Sastry C.P.V, Padmavathi M. Study of clinical and hematological profile in children with dengue fever in a teaching hospital in Telangana. Int J Pediatr Res. 2019;6(01):29-34.doi:10.17511/ijpr.2019.i01.05 